\title{
Clinical significance of delayed or slow graft function in kidney transplantation recipients
}

\author{
Jeongin Song ${ }^{1}$, Sehyun Jeong ${ }^{1}$, Sangil Min², Jongwon $\mathrm{Ha}^{2}$, Yon Su Kim${ }^{1}$, Jeong Pyo Lee ${ }^{1}$, Jong Cheol Jeong ${ }^{1}$, Hajeong Lee \\ ${ }^{1}$ Department of Internal Medicine, Seoul National University Hospital, Seoul, Korea \\ ${ }^{2}$ Department of Surgery, Seoul National University Hospital, Seoul, Korea
}

Background: With increasing total number of patients who receive expanded criteria donor kidneys, it is important to maintain the function of kidney grafts.

Methods: Retrospective medical records of kidney transplantation (KT) recipients from 2010 to 2021 in three hospitals were reviewed and analyzed. Patients were divided into three groups according to the degree of kidney function recovery after KT. Delayed graft function (DGF), slow graft function (SGF), and immediate graft function (IGF) were defined as the need for dialysis, serum creatinine levels $\geq 2.0 \mathrm{mg} / \mathrm{dL}$ but no need for dialysis, and serum creatinine levels $<2.0 \mathrm{mg} / \mathrm{dL}$ at 1 week after $\mathrm{KT}$, respectively.

Results: Among a total of 1,899 KT recipients, 73 DGF (3.8\%) and 137 SGF (7.2\%) were shown. DGF and SGF were developed more common in older recipient. Also, they tended to occur in cases of older donor age, male, deceased donor $\mathrm{KT}$, longer dialysis duration, and longer cold and warm ischemia time. Interestingly, DGF and SGF tended to increase recently. In the logistic regression analysis, older donor age, deceased donor, recent transplantation (since 2014) remained independent risk factors for both DGF and SGF compared with IGF. The most common cause of DGF was acute T-cell-mediated rejection and most of which were recovered after treatments such as rituximab, immunoglobulin and plasma exchange. During an average of 5 years of follow-up periods, 94 cases of graft failure and 40 cases of death were developed. DGF and IGF elevated graft failure risk as 3.0 (95\% confidence interval [Cl], 4.4-13.3; $\mathrm{P}=0.004)$ and $1.0(95 \% \mathrm{Cl}, 0.53-1.9 ; \mathrm{P}=0.983)$ times than IGF, respectively. There were no significant differences in mortality among three groups.

Conclusions: In this multicenter retrospective analysis, we found increasing tendency of DGF in KT recipients. Different from SGF, DGF showed worse graft outcomes than IGF although they shared several risk factors. Further clinical dissection should be warranted to improve graft outcomes because DGF seems to be more common in recent years.

Corresponding author: Hajeong Lee

E-mail:mdhjlee@gmail.com

(c) The Korean Society for Transplantation

This is an Open Access article distributed under the terms of the Creative Commons Attribution Non-Commercial License (http://creativecommons.org/licenses/by-nc/4.0/) which permits unrestricted non-commercial use, distribution, and reproduction in any medium, provided the original work is properly cited. 